\title{
Ciphertext Only Attack for One-way function of the MAP using One Ciphertext
}

\author{
Yukiyasu TSUNOO Eiji OKAMOTO Tomohiko UYEMATSU \\ School of Information Science \\ Japan Advanced Institute of Science and Technology \\ 15 Asahidai Tatsunokuchi Nomi Ishikawa, 923-12, Japan \\ Tel.:+81-761-51-1287, Fax.:+81-761-51-1338, Email:okamoto@jaist.ac.jp
}

\begin{abstract}
FEAL-N proposed by NTT is an $N$-round block cipher which is well suited for a fast software execution. We research the method which improves FEAL algorithm against cryptanalysis based on the careful analysis of data randomizer. Since the round function $f$ used as data randomizer has an inverse function $\mathrm{f}^{-1}$, we have already shown that there exists a key-independent relation between plaintext and ciphertext.

In this paper, we apply our attack to the ciphertext only attack for one-way function of the MAP using only one ciphertext. Computer implementation of the proposed attack shows that the improvement of the computational complexity to obtain the plaintext is decreased and given by $O\left(2^{32}\right)$.
\end{abstract}

\section{Introduction}

FEAL-N proposed by NTT is a $N$-round block cipher which is well suited for a fast software implementation on 8 or 16 bit microprocessors. FEAL is a DESlike secret-key cryptosystem ${ }^{[1][2]}$. There are many kinds of attacks of FEAL, such as a differential attack ${ }^{[3]}$, a statistical attack ${ }^{[4]}$, an attack using a linear approximation of S-boxes ${ }^{[5]}$, an attack using bit-equations of data randomizer ${ }^{[6]}$ and so on ${ }^{[7]}$. We research the method which improves FEAL algorithm against cryptanalysis by based on the careful analysis of data randomizer. We have studied an attack that employs an inverse function $\mathrm{f}^{-1}$ of the intermediate message blocks, where $f$ is a round function of FEAL. Our proposed attack is divided into two steps. First step is to search out intermediate message blocks using a key-independent relation of a FEAL data randomizer between ciphertext blocks and corresponding plaintext blocks. Second step is to calculate the key using the above inverse function $\mathrm{f}^{-1}$ of the intermediate message blocks.

In this paper, we apply our method to the ciphertext only attack for a oneway function that employs the mapping function called MAP ${ }^{[1]}$. The algorithm of MAP function is very similar to that of FEAL data randomizer in the shape of the round function $f$. By virtue of the simplification of the MAP algorithm, fairly simple related equations between ciphertext blocks and corresponding plaintext blocks are given. Only one 64-bit ciphertext is needed to calculate the corresponding plaintext and its computational complexity is $O\left(2^{32}\right)$. This implies 
that the computational complexity to obtain the plane text is decreased and given by $O\left(2^{32}\right)$.

The contents of this paper is given in three parts; first part relates to the specification of one-way function of the MAP, second part concerns our proposed ciphertext only attack and final part describes concluding remarks.

\section{One-way function of the MAP}

The one-way function of the MAP is a 4-round Feistel block cipher operating on 64-bit plaintext and ciphertext blocks. A round function depends on the output of the previous round only and has nothing to do with enciphering/deciphering key. The one-way function of the MAP is shown in Fig.1.

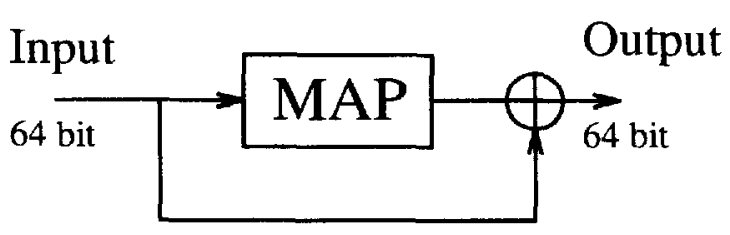

Fig. 1. One-way function of the MAP

The MAP algorithm is based on iterating a round function $f$ four times. The round function $f$ is based on nonlinear S-box functions. The MAP algorithm, $S$-box and $f$ functions are defined in the following sections.

\subsection{MAP Algorithm}

The MAP algorithm is shown in Fig.2. In the mapping process, a 64-bit input block is partitioned into eight 8 -bit subblocks, and transformed into eight 8-bit output subblocks. The MAP algorithm performs as follows:

Suppose we compute a 64-bit input block. At first, we split the input block into left and right halves of 32-bit long, and compute the exclusiveor (hereafter exclusive-or is referred as XOR) of the 32-bit left half and the 32-bit right half as shown in Fig.2. Second, we compute the XOR of the 32-bit left half and a 32-bit random number calculated by function $\mathrm{f}$ shown in Fig.3. We then interchange both the 32 -bit left and the 32 -bit

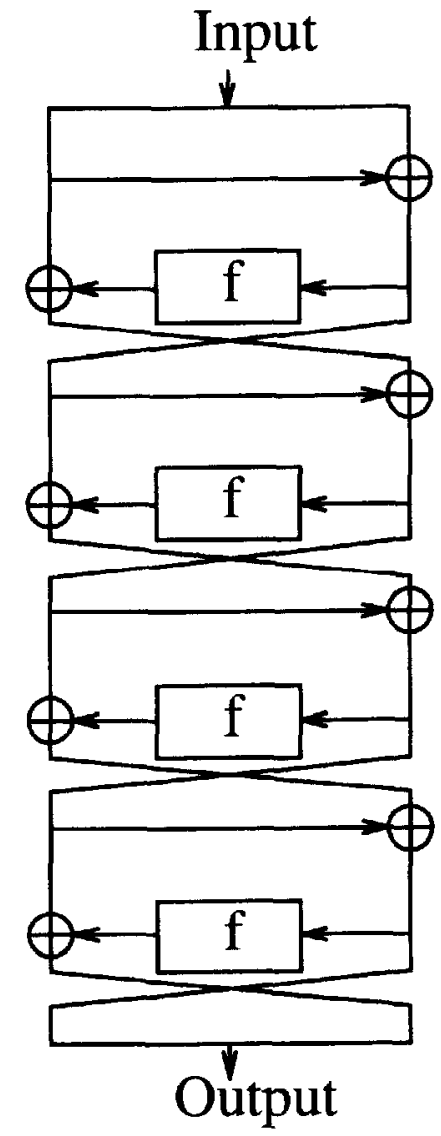

Fig. 2. MAP algorithm 
right halves. In order to get a 64-bit output block, we perform these processes four times. Obviously, this MAP algorithm has the inverse algorithm $\mathrm{MAP}^{-1}$, because we can perform the same procedure above in reverse. Since the computational complexity of calculating output blocks ( depicted as Output ) from input blocks (depicted as Input) shown in Fig.2 is equal to that of calculating input blocks from output blocks, this MAP algorithm cannot be regarded as a one-way function without any changes. Thus, the one-way function using MAP algorithm shown in Fig. 1 may be considered as a 64-bit block cipher based on a 64bit key where the key is a 64-bit input block. Thus, in Fig.1, the computational complexity of calculating input blocks from output blocks is given by $O\left(2^{64}\right)$.

\subsection{Function S-BOX}

The S-box function has two 1-byte inputs, $x_{1}$ and $x_{2}$, and outputs one 1-byte block, such that

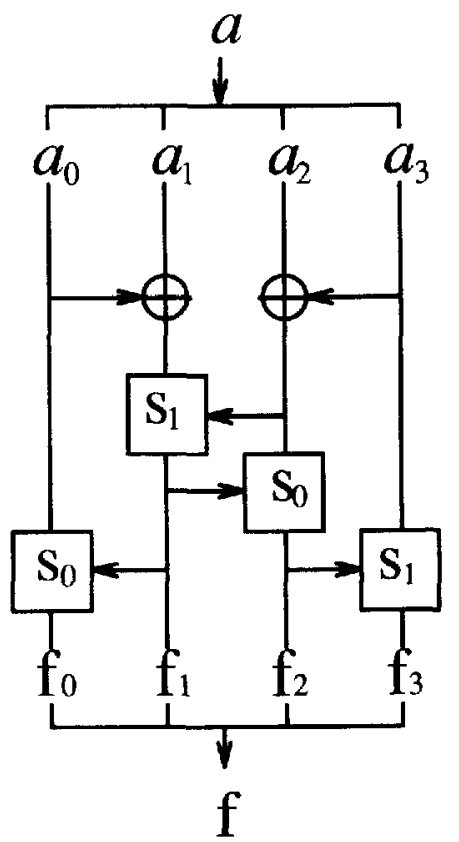

Fig. 3. Function $f$

$$
\begin{aligned}
S_{d}\left(x_{1}, x_{2}\right) & =\operatorname{rot}_{2}\left(x_{3}\right) \text { and } \\
x_{3} & =x_{1}+x_{2}+d \bmod 256
\end{aligned}
$$

where $d \in\{0,1\}$, and $\operatorname{rot}_{2}\left(x_{3}\right)$ denotes a 2 -bit left rotation on 8 -bit data $x_{3}$.

\subsection{Function $\mathbf{f}$}

The round function $f$ has one 4 -byte input and outputs one 4-byte block. Let $a=\left(a_{0}, a_{1}, a_{2}, a_{3}\right)$ be a 4 -byte input block and $f=\left(f_{0}, f_{1}, f_{2}, f_{3}\right)$ be a 4-byte output block. Then, the function $f$ is defined as the following equations:

$$
\begin{aligned}
& f_{1}=S_{1}\left(a_{1} \oplus a_{0}, a_{2} \oplus a_{3}\right) \\
& f_{2}=S_{0}\left(f_{1}, a_{2} \oplus a_{3}\right) \\
& f_{0}=S_{0}\left(a_{0}, f_{1}\right) \\
& f_{3}=S_{1}\left(a_{3}, f_{2}\right)
\end{aligned}
$$

where symbol $\oplus$ denotes the XOR operation. Function $f$ can be illustrated in Fig.3. ( In FEAL algorithm, equation $f_{1}=S_{1}\left(a_{1} \oplus a_{0} \oplus \beta_{0}, a_{2} \oplus a_{3} \oplus \beta_{1}\right)$ and $f_{2}=S_{0}\left(f_{1}, a_{2} \oplus a_{3} \oplus \beta_{1}\right)$, where $\beta_{0}$ and $\beta_{1}$ denote a 8 -bit key respectively, are applied to definitions of $f_{1}$ and $f_{2}$ instead of equation (2.3) and (2.4)). 


\section{Proposed Ciphertext Only Attack}

We consider a ciphertext-only attack. In general, assumption of cryptography, the attacker can access to the plaintext/ciphertext transmitted over the insecure channel. According to the information obtained by the attacker, attacks are usually classified into four types:

(1) ciphertext-only attack,

(2) known-plaintext attack,

(3) chosen-plaintext attack and

(4) chosen-ciphertext attack.

In this classification, the ciphertext-only attack includes the other types of $\operatorname{attack}^{[11]}$; that is, if a ciphertext is decrypted by the ciphertext-only attack, then other attacks can decrypt the same ciphertext without any additional information. The ciphertext-only attack means that the attacker has no information except sufficient number of arbitrary ciphertexts. In our proposed attack, if only one ciphertext is given, then we will manage to compute plaintext depending on that ciphertext.

\subsection{Outline of Attack}

As shown in 2.1, as for the one-way function of the MAP shown in Fig.1, we can compute input blocks from output blocks with the computational complexity of $O\left(2^{64}\right)$. But $O\left(2^{64}\right)$ of computational complexity means the intractable numerical problem and input blocks may not be computed from output blocks.

Our proposed attack needs the computational complexity of the size $O\left(2^{32}\right)$ and is divided into three steps.

(1) Simplify the MAP algorithm,

(2) Find out the relation between plaintext and ciphertext

(3) Compute plaintext by means of the relation (2).

The computation of step (3) can be performed by a brute force attack with computational complexity of $O\left(2^{32}\right)$.

\subsection{Simplification of the MAP Algorithm}

In this subsection, we simplify the MAP algorithm shown in Fig.2

Fig. 4 shows the equivalent algorithm to Fig.2. In Fig.4, both the second round and the forth round of function $f$ are applied in the opposite direction, instead of the interchanging between the 32-bit left half and the 32-bit right half calculated by previous rounds of function $f$. In what follows, the 32-bit left half and the 32-bit right half of Input blocks are described as $A_{0}$ and $B_{0}$, the 32-bit left half and the 32 -bit right half of blocks calculated by the second round (hereafter call these blocks intermediate message) are described as $A_{2}$ and $B_{2}$ and the 32-bit left half and the 32-bit right half of Output blocks are described as $A_{4}$ and $B_{4}$. 


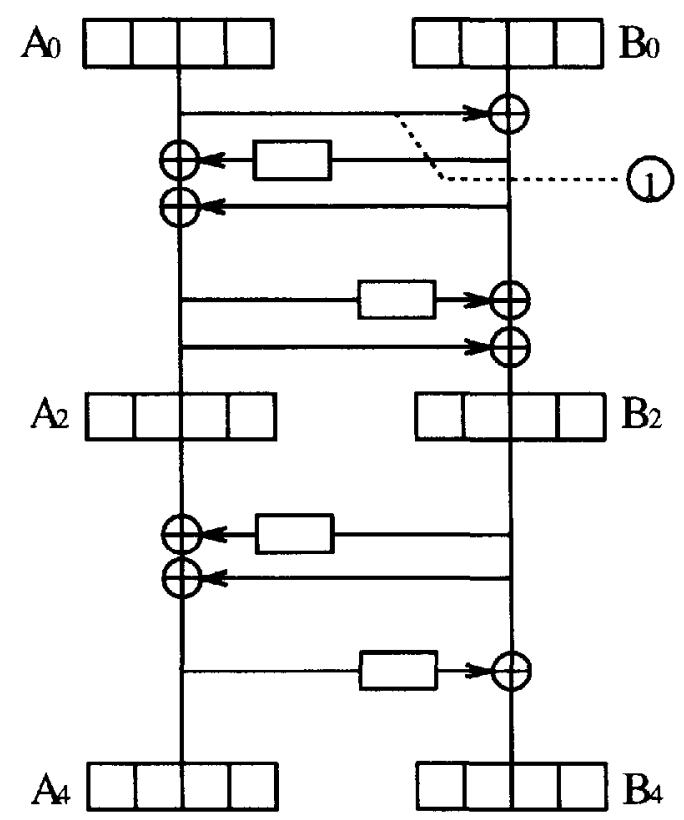

Fig. 4. 1st Simplification

We also define in following Figures, $A_{i j}$ and $B_{i j},(i=0,2,4),(j=0,1,2,3)$, denote the 1-byte block defined by following equations: $A_{i}=\left(A_{i 0}, A_{i 1}, A_{i 2}, A_{i 3}\right), B_{i}=\left(B_{i 0}, B_{i 1}, B_{i 2}, B_{i 3}\right)$.

The simplification procedure of the MAP algorithm is shown below.

(1) In Fig.4, the first XOR at the circle number one (at(1) with a dotted line ) can be omitted, if input blocks are given by $\left(A_{0}, A_{0} \oplus B_{0}\right)$ instead of ( $\left.A_{0}, B_{0}\right)$.

Thus, Fig. 4 and Fig. 5 are equivalent.

(2) In Fig.5, without affecting of all the value of $A_{i}$ and $B_{i},(i=0,2,4)$, the XOR just after the first round function $f$ can be moved just before the first round one, and the XOR just after the third round function $f$ can also be moved just before the third round one, (at (2) and (3) with a dotted curve in Fig.6, respectively).

Thus, Fig.5 and Fig. 6 are equivalent.

(3) In Fig.6, the XOR after the movement depicted by the circle number two ( at (2) with a dotted curve ) can be omitted, if input blocks are given by ( $\left.B_{0}, A_{0} \oplus B_{0}\right)$ instead of $\left(A_{0}, A_{0} \oplus B_{0}\right)$.

Thus, Fig. 6 and Fig.7 are equivalent.

(4) In Fig.7, let us estimate the value of the intermediate messages indicated by the circle number four, five, six and seven ( see (4), (5), (6) and (7) with a dotted message blocks, respectively ). The value of intermediate message at 


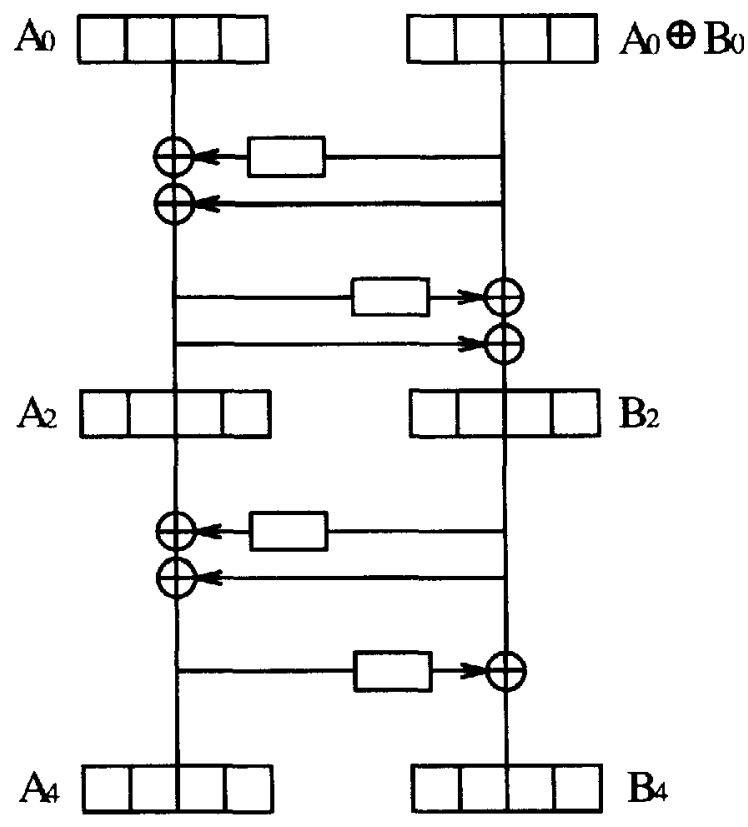

Fig. 5. 2nd Simplification

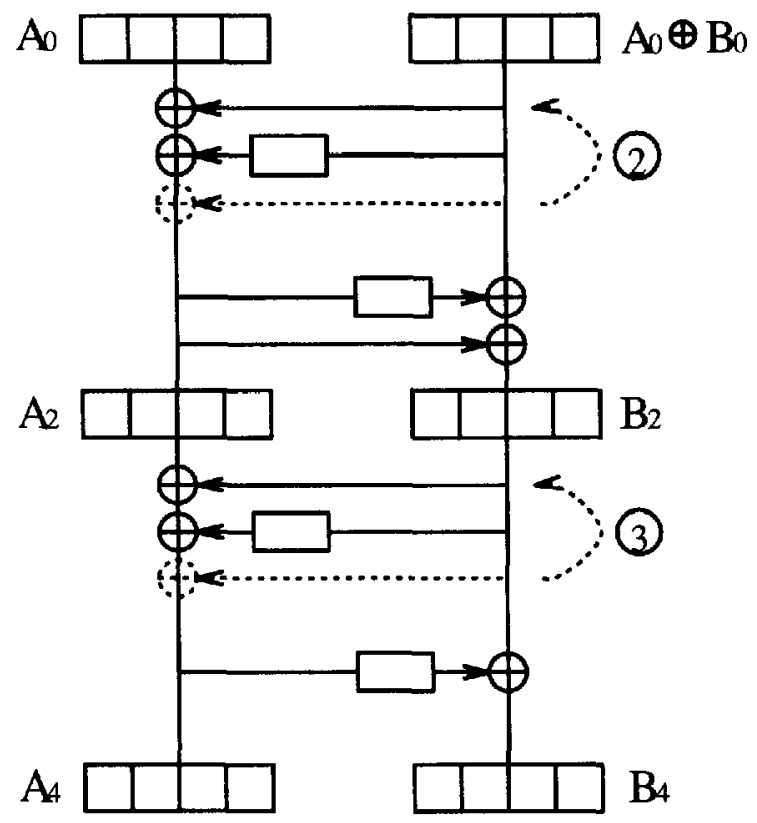

Fig. 6. 3rd Simplification 


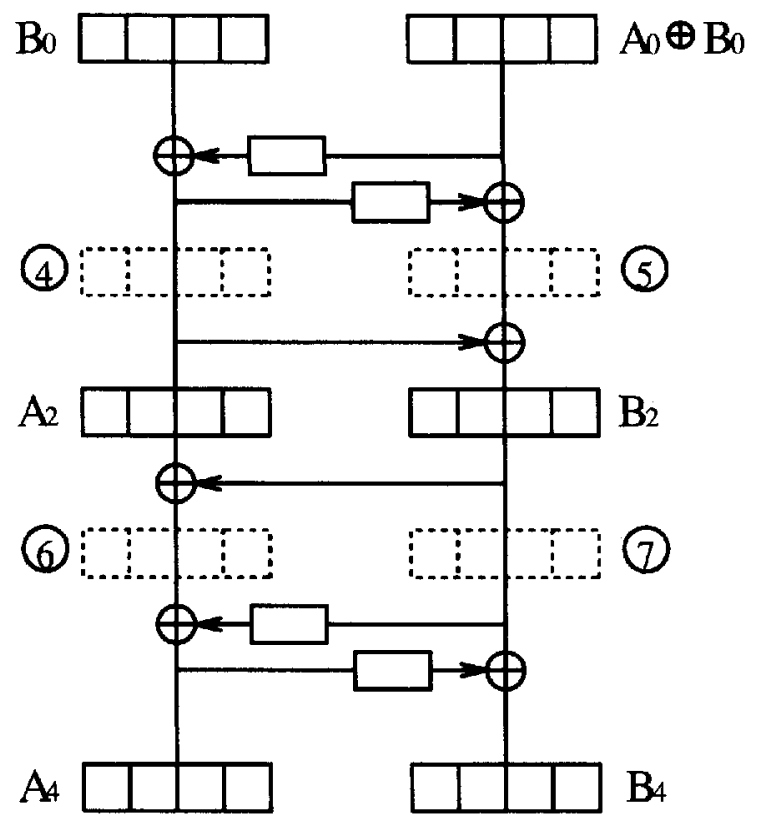

Fig. 7. 4th Simplification
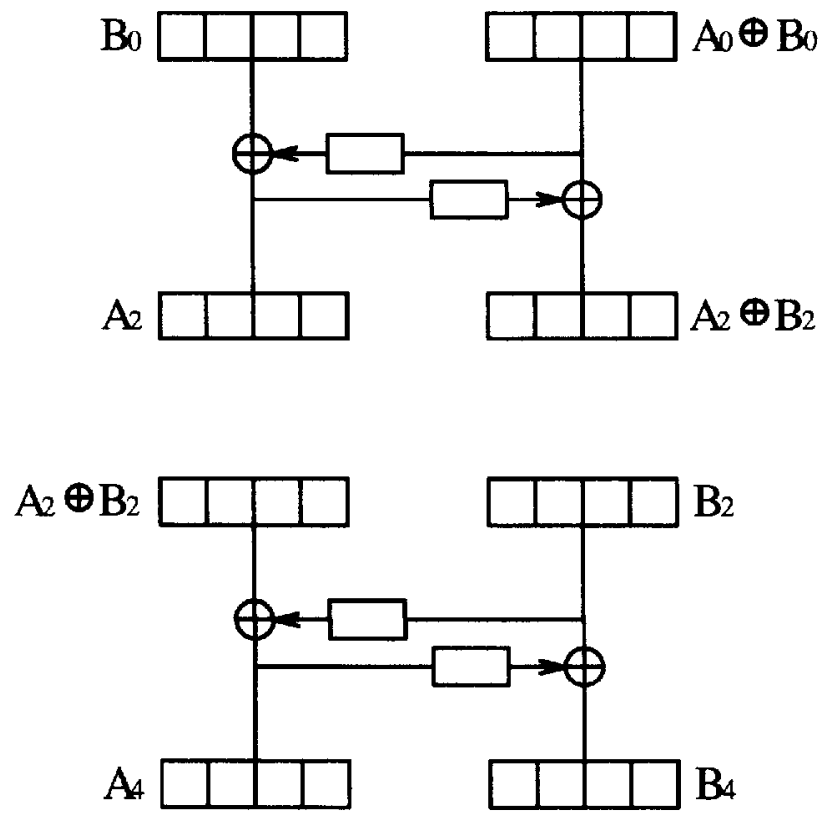

Fig. 8. 5th Simplification 


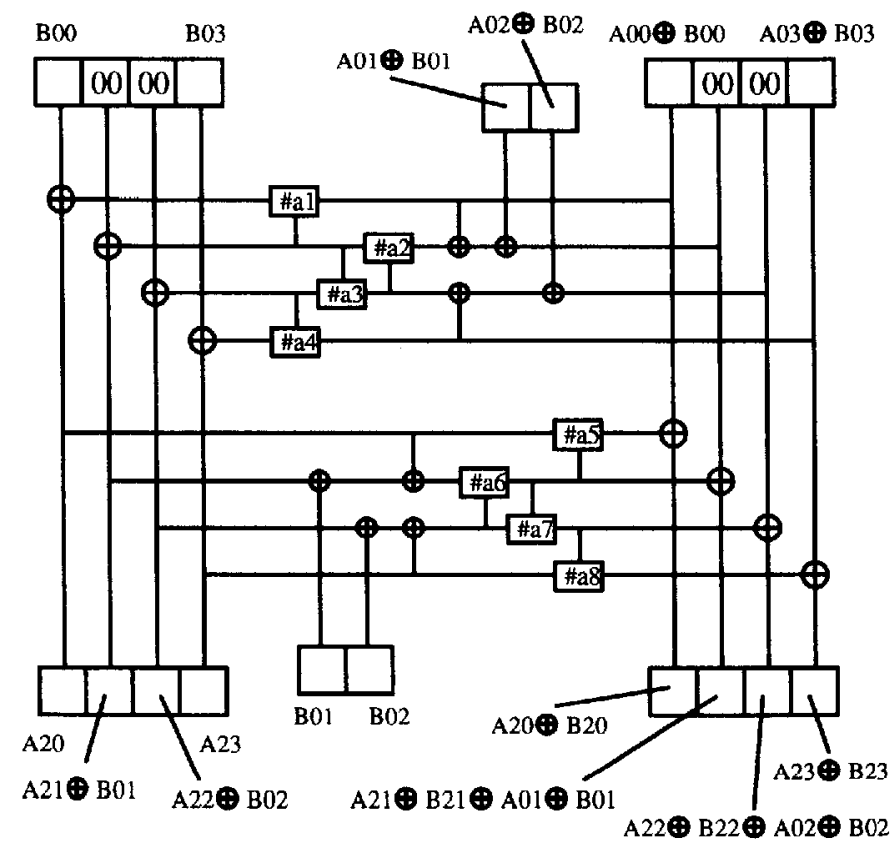

Fig. 9. 6th Simplification by S-Box (a)

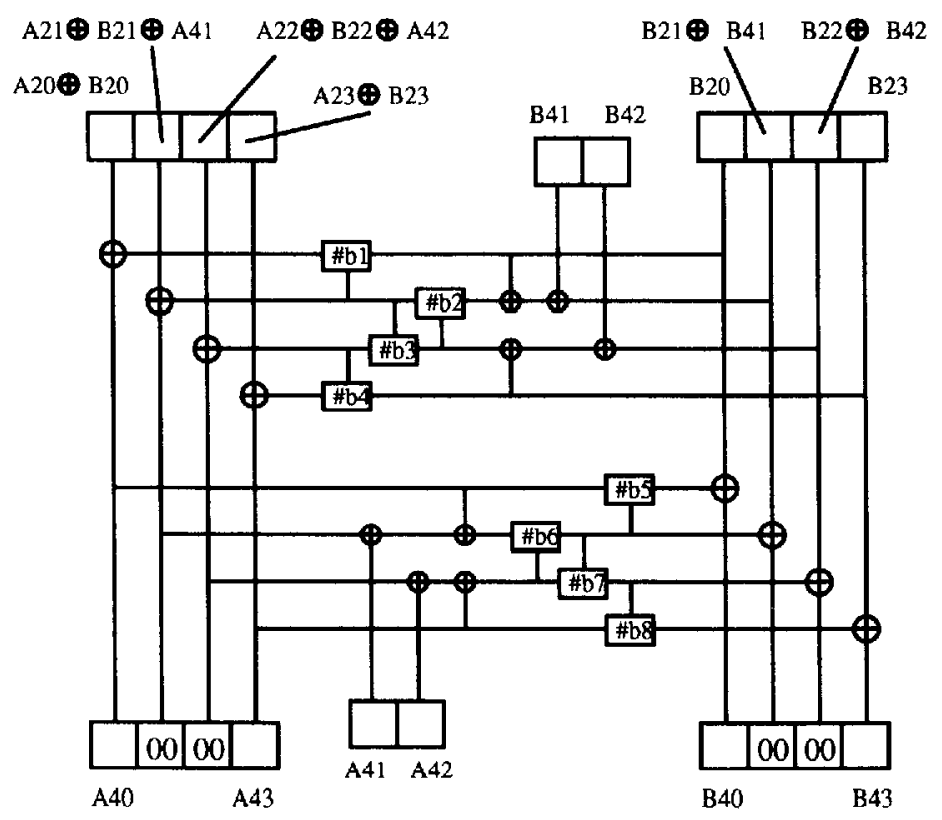

Fig. 10. 6th Simplification by S-Box (b) 
(4) is equivalent to the value of $A_{2}$, the value of intermediate message at (7) is equivalent to the value of $B_{2}$, the value of intermediate message at(5) and at(6) are equivalent to the value of $\left(A_{2} \oplus B_{2}\right)$.

Thus, Fig.7 and Fig. 8 are equivalent.

(5) Now, let a new mechanism introduce into Fig.8. The new mechanism exchanges the value of the 1-byte blocks at $B_{01}, B_{02}, A_{01} \oplus B_{01}, A_{02} \oplus B_{02}$, $A_{41}, A_{42}, B_{41}$ and $B_{42}$ with the value of " 00 ". In order to maintain the value of these 1-byte blocks, we introduce two XORs into each function $\mathrm{f}$ shown in the pair of Fig.9 and Fig.10. Fig.8 is equivalent to the pair of Fig.9 and Fig.10. Let notice as follows:

In Fig.9, the new XOR function of $\left(A_{01} \oplus B_{01}, A_{02} \oplus B_{02}\right)$ and $\left(B_{01}, B_{02}\right)$ are introduced into the input blocks of function S-Box denoted by (\#a2,\#a3) and (\#a6,\#a7) and the value of the 1 -byte blocks at $\left(A_{21}, A_{22}\right)$ and $\left(A_{21} \oplus B_{21}, A_{22} \oplus B_{22}\right)$ are replaced by $\left(A_{21} \oplus B_{01}, A_{22} \oplus B_{02}\right)$ and $\left(A_{21} \oplus B_{21} \oplus A_{01} \oplus B_{01}, A_{22} \oplus B_{22} \oplus A_{02} \oplus B_{02}\right)$, respectively. Also, in Fig. 10, new XOR function of $\left(B_{41}, B_{42}\right)$ and $\left(A_{41}, A_{42}\right)$ are introduced into the input blocks of function S-Box denoted by $(\# b 2, \# b 3)$ and $(\# b 6, \# b 7)$ and the value of the 1 -byte blocks at $\left(A_{21} \oplus B_{21}, A_{22} \oplus B_{22}\right)$ and $\left(B_{21}\right.$, $\left.B_{22}\right)$ are replaced by $\left(A_{21} \oplus B_{21} \oplus A_{41}, A_{22} \oplus B_{22} \oplus A_{42}\right)$ and $\left(B_{21} \oplus B_{41}\right.$, $\left.B_{22} \oplus B_{42}\right)$, respectively.

According to the above simplification procedure, Fig. 4 is equivalent to the pair of Fig. 9 and Fig.10. Therefore, Fig.2 can be simplified into Fig.9 and Fig.10.

\subsection{Calculation of plaintext}

In this subsection, we give some equations that can compute input blocks from output blocks shown in Fig.1. By using these equations, the plaintext $\left(\left(A_{00}, A_{01}\right.\right.$, $\left.\left.A_{02}, A_{03}\right),\left(B_{00}, B_{01}, B_{02}, B_{03}\right)\right)$ can be computed from a given ciphertext $\left(C_{0}\right.$, $\left.C_{1}, C_{2}, C_{3}, C_{4}, C_{5}, C_{6}, C_{7}\right)$.

From now on, let us call the one-way function of the MAP in Fig.1 the target function and call the MAP algorithm in Fig.2 simply the MAP. Let us consider the computational complexity of ciphertext only attack for the target function, which depends on the computational complexity of the brute force attack. The computational complexity of this attack can be described as the number of tried decryptions. Seeing Fig.4, obviously, the MAP does not have the one-way property, because the value of both $A_{0}$ blocks and $B_{0}$ blocks can be easily calculate from the value of both $A_{4}$ blocks and $B_{4}$ blocks. Seeing Fig.1, however, the target function seems to be practically secure against brute force attack, because the value of output blocks are given by the XOR of the input blocks (that is $A_{0}$ and $B_{0}$ ) and the output of the MAP (that is $A_{4}$ and $B_{4}$ ). So, the computational complexity of the brute force attack for the target function seems to be given by $O\left(2^{64}\right)$. But, we show that the computational complexity to obtain input blocks(i.e. plaintext) from output blocks(i.e. ciphertext) can be decreased to $O\left(2^{32}\right)$ from $O\left(2^{64}\right)$. 
Let us recall equation(2.1) and (2.2) about function S-box. Any one of three values $x_{1}, x_{2}$ and $x_{3}$ can be determined by the other two values. From now on, this relationship must be taken into consideration.

(1) [ Relationship between ciphertext and plaintext ]

Let eight 1-byte blocks of ciphertext be $C_{0}, C_{1}, C_{2}, C_{3}, C_{4}, C_{5}, C_{6}$ and $C_{7}$. Then, the following equations is valid in Fig. 4, 5, 6, 7, 8, 9 and 10:

$$
\begin{aligned}
& A_{40}=C_{0} \oplus A_{00}, \\
& A_{41}=C_{1} \oplus A_{01}, \\
& A_{42}=C_{2} \oplus A_{02}, \\
& A_{43}=C_{3} \oplus A_{03}, \\
& B_{40}=C_{4} \oplus B_{00}, \\
& B_{41}=C_{5} \oplus B_{01}, \\
& B_{42}=C_{6} \oplus B_{02} \text { and } \\
& B_{43}=C_{7} \oplus B_{03} .
\end{aligned}
$$

Now, suppose four 1-byte blocks, i.e. $A_{00}, B_{00}, A_{20}$ and $B_{20}$, are given. Hereafter, $\operatorname{lot}_{2}(x)$ denotes a 2 -bit right rotation on 8 -bit data $x$, and \#ai and \#bi, $(i=1,2, \cdots, 8)$, denote the function S-Box in Fig.9 and Fig.10.

(2) [ Relation of function S-Box (\#a1, \#a5, \#b1, \#b5)]

According to each of the function S-Box denoted by \#a1, \#a5, \#b1 and \#b5, we can obtain the following equations, (3.9), (3.10),(3.11) and (3.12).

\#a1 : $A_{21} \oplus B_{01}=\operatorname{lot}_{2}\left(B_{00} \oplus A_{20}\right)-A_{00} \oplus B_{00}$

\#a5: $A_{21} \oplus B_{21} \oplus A_{01} \oplus B_{01}=\operatorname{lot}_{2}\left(A_{00} \oplus B_{00} \oplus A_{20} \oplus B_{20}\right)-A_{20}$

\#b1 : $A_{21} \oplus B_{21} \oplus C_{1} \oplus A_{01}=\operatorname{lot}_{2}\left(C_{0} \oplus A_{00} \oplus A_{20} \oplus B_{20}\right)-B_{20}$

\#b5: $B_{21} \oplus C_{5} \oplus B_{01}=\operatorname{lot}_{2}\left(B_{20} \oplus C_{4} \oplus B_{00}\right)-C_{0} \oplus A_{00}$

By equation (3.10) and (3.11) we obtain

$B_{01}=\left\{\operatorname{lot}_{2}\left(A_{00} \oplus B_{00} \oplus A_{20} \oplus B_{20}\right)-A_{20}\right\}$

$\oplus\left\{\operatorname{lot}_{2}\left(C_{0} \oplus A_{00} \oplus A_{20} \oplus B_{20}\right)-B_{20}\right\} \oplus C_{1}$

By (3.9)

$A_{21}=\left\{\operatorname{lot}_{2}\left(B_{00} \oplus A_{20}\right)-A_{00} \oplus B_{00}\right\} \oplus B_{01}$

By (3.12)

$B_{21}=\left\{\operatorname{lot}_{2}\left(B_{20} \oplus C_{4} \oplus B_{00}\right)-C_{0} \oplus A_{00}\right\} \oplus C_{5} \oplus B_{01}$

By (3.11)

$A_{01}=\left\{\operatorname{lot}_{2}\left(C_{0} \oplus A_{00} \oplus A_{20} \oplus B_{20}\right)-B_{20}\right\} \oplus A_{21} \oplus B_{21} \oplus C_{1}$

Thus, we obtained four 1-byte blocks, i.e. $A_{01}, B_{01}, A_{21}$ and $B_{21}$. 
(3) [Relation of function S-Box (\#a2, \#a3, \#a6, \#a7, \#b2, \#b3, \#b6, \#b7)]

According to each of the function S-Box denoted by \#a2, \#a3, \#a6, \#a7, \#b2, \#b3, \#b6 and \#b7, we can obtain the following equations, (3.17), (3.18), (3.19), (3.20), (3.21), (3.22),(3.23) and (3.24).

\#a2 : $\left\{A_{02} \oplus B_{02}\right\} \oplus A_{03} \oplus B_{03}=\operatorname{lot}_{2}\left(A_{21} \oplus B_{01}\right)-A_{00} \oplus B_{00} \oplus A_{01} \oplus B_{01}-1$

$\#$ a3 : $\left\{A_{02} \oplus B_{02}\right\} \oplus A_{03} \oplus B_{03}=\operatorname{lot}_{2}\left(A_{22} \oplus B_{02}\right)-A_{21} \oplus B_{01}$

\#a6 : $\left\{B_{02}\right\} \oplus A_{22} \oplus B_{02} \oplus A_{23}$

$=\operatorname{lot}_{2}\left(A_{21} \oplus B_{21} \oplus A_{01} \oplus B_{01}\right)-\left\{B_{01}\right\} \oplus A_{20} \oplus A_{21} \oplus B_{01}-1$

\#a $7:\left\{B_{02}\right\} \oplus A_{22} \oplus B_{02} \oplus A_{23}$

$=\operatorname{lot}_{2}\left(A_{22} \oplus B_{22} \oplus A_{02} \oplus B_{02}\right)-A_{21} \oplus B_{21} \oplus A_{01} \oplus B_{01}$

\#b2: $\left\{C_{6} \oplus B_{02}\right\} \oplus B_{22} \oplus C_{6} \oplus B_{02} \oplus B_{23}$

$=\operatorname{lot}_{2}\left(A_{21} \oplus B_{21} \oplus C_{1} \oplus A_{01}\right)-\left\{C_{5} \oplus B_{01}\right\} \oplus B_{20} \oplus B_{21} \oplus C_{5} \oplus B_{01}-1$

\#b3: $\left\{C_{6} \oplus B_{02}\right\} \oplus B_{22} \oplus C_{6} \oplus B_{02} \oplus B_{23}$

$=\operatorname{lot}_{2}\left(A_{22} \oplus B_{22} \oplus C_{2} \oplus A_{02}\right)-A_{21} \oplus B_{21} \oplus C_{1} \oplus A_{01}$

\#b6: $\left\{C_{2} \oplus A_{02}\right\} \oplus C_{3} \oplus A_{03}=\operatorname{lot}_{2}\left(B_{21} \oplus C_{5} \oplus B_{01}\right)-C_{0} \oplus A_{00} \oplus C_{1} \oplus A_{01}-1$

\#b7: $\left\{C_{2} \oplus A_{02}\right\} \oplus C_{3} \oplus A_{03}=\operatorname{lot}_{2}\left(B_{22} \oplus C_{6} \oplus B_{02}\right)-B_{21} \oplus C_{5} \oplus B_{01}$

By the combinations of equations ((3.17), (3.18)), ((3.19), (3.20)), ((3.21), $(3.22))$ and $((3.23),(3.24))$, we can obtain the following equations.

$\#$ a2, \#a3 : $A_{22} \oplus B_{02}$

$=\operatorname{rot}_{2}\left(\operatorname{lot}_{2}\left(A_{21} \oplus B_{01}\right)-A_{00} \oplus B_{00} \oplus A_{01} \oplus B_{01}-1+A_{21} \oplus B_{01}\right)$

\#a6, \#a7 : $A_{22} \oplus B_{22} \oplus A_{02} \oplus B_{02}$

$=\operatorname{rot}_{2}\left(\operatorname{lot}_{2}\left(A_{21} \oplus B_{21} \oplus A_{01} \oplus B_{01}\right)-A_{20} \oplus A_{21}-1+A_{21} \oplus B_{21} \oplus A_{01} \oplus B_{01}\right)$

\#b2, \#b3: $A_{22} \oplus B_{22} \oplus A_{02}$

$=C_{2} \oplus \operatorname{rot}_{2}\left(\operatorname{lot}_{2}\left(A_{21} \oplus B_{21} \oplus C_{1} \oplus A_{01}\right)\right.$

$\left.-B_{20} \oplus B_{21}-1+A_{21} \oplus B_{21} \oplus C_{1} \oplus A_{01}\right)$

\#b6, \#b7 : $B_{22} \oplus B_{02}$

$=C_{6} \oplus \operatorname{rot}_{2}\left(\operatorname{lot}_{2}\left(B_{21} \oplus C_{5} \oplus B_{01}\right)-C_{0}\right.$

$\left.\oplus A_{00} \oplus C_{1} \oplus A_{01}-1+B_{21} \oplus C_{5} \oplus B_{01}\right)$

By equation (3.26) and (3.27) we obtain

$B_{02}=\left\{\operatorname{rot}_{2}\left(\operatorname{lot}_{2}\left(A_{21} \oplus B_{21} \oplus A_{01} \oplus B_{01}\right)-A_{20} \oplus A_{21}-1+A_{21} \oplus B_{21} \oplus A_{01} \oplus B_{01}\right)\right\}$

$\oplus\left\{C_{2} \oplus \operatorname{rot}_{2}\left(\operatorname{lot}_{2}\left(A_{21} \oplus B_{21} \oplus C_{1} \oplus A_{01}\right)\right.\right.$

$\left.\left.-B_{20} \oplus B_{21}-1+A_{21} \oplus B_{21} \oplus C_{1} \oplus A_{01}\right)\right\}$

By $(3.25)$

$A_{22}=\left\{\operatorname{rot}_{2}\left(\operatorname{lot}_{2}\left(A_{21} \oplus B_{01}\right)-A_{00} \oplus B_{00} \oplus A_{01} \oplus B_{01}-1+A_{21} \oplus B_{01}\right)\right\} \oplus B_{02}$ 
By (3.28)

$B_{22}=\left\{C_{6} \oplus \operatorname{rot}_{2}\left(\operatorname{lot}_{2}\left(B_{21} \oplus C_{5} \oplus B_{01}\right)\right.\right.$

$\left.\left.-C_{0} \oplus A_{00} \oplus C_{1} \oplus A_{01}-1+B_{21} \oplus C_{5} \oplus B_{01}\right)\right\} \oplus B_{02}$

By (3.27)

$A_{02}=\left\{C_{2} \oplus \operatorname{rot}_{2}\left(\operatorname{lot}_{2}\left(A_{21} \oplus B_{21} \oplus C_{1} \oplus A_{01}\right)\right.\right.$

$\left.\left.-B_{20} \oplus B_{21}-1+A_{21} \oplus B_{21} \oplus C_{1} \oplus A_{01}\right)\right\} \oplus A_{22} \oplus B_{22}$

Thus, we obtained four 1 -byte blocks, i.e. $A_{02}, B_{02}, A_{22}$ and $B_{22}$.

By (3.24)

$A_{03}=\left\{\operatorname{lot}_{2}\left(B_{22} \oplus C_{6} \oplus B_{02}\right)-B_{21} \oplus C_{5} \oplus B_{01}\right\} \oplus C_{2} \oplus A_{02} \oplus C_{3}$

By (3.22)

$B_{23}=\left\{l_{\text {oot }}\left(A_{22} \oplus B_{22} \oplus C_{2} \oplus A_{02}\right)-A_{21} \oplus B_{21} \oplus C_{1} \oplus A_{01}\right\} \oplus B_{22}$

By (3.20)

$A_{23}=\left\{\operatorname{lot}_{2}\left(A_{22} \oplus B_{22} \oplus A_{02} \oplus B_{02}\right)-A_{21} \oplus B_{21} \oplus A_{01} \oplus B_{01}\right\} \oplus A_{22}$

By (3.18)

$B_{03}=\left\{\operatorname{lot}_{2}\left(A_{22} \oplus B_{02}\right)-A_{21} \oplus B_{01}\right\} \oplus A_{02} \oplus B_{02} \oplus A_{03}$

We obtained four 1-byte blocks, i.e. $A_{03}, B_{03}, A_{23}$ and $B_{23}$.

(4) [ Relation of function S-Box (\#a4,\#a8,\#b4,\#b8)]

According to each of the function S-Box denoted by \#a4, \#a8, \#b4 and \#b8, the value of $A_{02}, B_{02}, A_{22}, B_{22}, A_{03}, B_{03}, A_{23}$ and $B_{23}$ must satisfy the following four equations, (3.37), (3.38),(3.39) and (3.40).

\#a4: $A_{22} \oplus B_{02}=\operatorname{lot}_{2}\left(A_{23} \oplus B_{03}\right)-A_{03} \oplus B_{03}-1$

\#a8: $A_{22} \oplus B_{22} \oplus A_{02} \oplus B_{02}=\operatorname{lot}_{2}\left(A_{23} \oplus B_{23} \oplus A_{03} \oplus B_{03}\right)-A_{23}-1$

\#b4: $A_{22} \oplus B_{22} \oplus C_{2} \oplus A_{02}=\operatorname{lot}_{2}\left(C_{3} \oplus A_{03} \oplus A_{23} \oplus B_{23}\right)-B_{23}-1$

\#b8: $B_{22} \oplus C_{6} \oplus B_{02}=\operatorname{lot}_{2}\left(B_{23} \oplus C_{7} \oplus B_{03}\right)-C_{3} \oplus A_{03}-1$

This means that these equations are used for checks on the correctness of given value of $A_{00}, B_{00}, A_{20}, B_{20}$.

According to the above discussion, twelve 1-byte blocks, i.e. $A_{01}, A_{02}, A_{03}$, $B_{01}, B_{02}, B_{03}, A_{21}, A_{22}, A_{23}, B_{21}, B_{22}$ and $B_{23}$, can be computed when one ciphertext $\left(C_{0}, C_{1}, C_{2}, C_{3}, C_{4}, C_{5}, C_{6}\right.$ and $\left.C_{7}\right)$ is given, on the assumption that four 1-byte blocks $\left(A_{00}, B_{00}, A_{20}\right.$ and $\left.B_{20}\right)$ are given.

Therefore, taking the equations $(3.1-3.8)$ into consideration, when one ciphertext $\left(C_{i}\right),(i=0, \cdots, 7)$, is given and that four 1-byte blocks ( $A_{00}, B_{00}, A_{20}$ and $B_{20}$ ) are presumed, we can compute twenty four 1-byte blocks, i.e. $A_{00}, A_{01}$, $A_{02}, A_{03}, B_{00}, B_{01}, B_{02}, B_{03}, A_{20}, A_{21}, A_{22}, A_{23}, B_{20}, B_{21}, B_{22}, B_{23}, A_{40}, A_{41}$, $A_{42}, A_{43}, B_{40}, B_{41}, B_{42}$ and $B_{43}$, that satisfy the relation shown in Fig.9 and Fig. 10. 


\subsection{Example of computation}

We perform the calculation with the ciphertext given as:

$$
\left(C_{i}\right)=(d a, 8 a, b 9, d b, 34, d 1,59,94), \quad(i=0, \cdots, 7)
$$

under relationship of the equations shown in the previous subsection3.3.

Three patterns of the computed plaintext are obtained as follows:

$$
\begin{aligned}
\left(A_{0}, B_{0}\right) & =(48,41,53,48,44,41,54,41) \\
\left(A_{2}, B_{2}\right) & =(a 5,2 d, b d, 8 e, f 7, d f, 2 e, 0 b) \\
\left(A_{4}, B_{4}\right) & =(92, c b, e a, 93,70,90,0 d, d 5) \\
\left(A_{0}, B_{0}\right) & =(4 f, 1 b, 01,40,69,2 b, 12,08) \\
\left(A_{2}, B_{2}\right) & =(d 6, e 2,82,6 f, 89,5 a, 44,32) \\
\left(A_{4}, B_{4}\right) & =(95,91, b 8,9 b, 5 d, f a, 4 b, 9 c) \\
& \text { and } \\
\left(A_{0}, B_{0}\right) & =(55,20, a c, 80,37, f a, 48, d 7) \\
\left(A_{2}, B_{2}\right) & =(7 b, 4 b, d 9,70,82, f a, 6 d, 20) \\
\left(A_{4}, B_{4}\right) & =(8 f, a a, 15,5 b, 03,2 b, 11,43) .
\end{aligned}
$$

It took 15.7 hours to compute the above case, with $\mathrm{C}$ program on a workstation (SUN-SPARCstation 2).

The obtained plaintext is not unique. But only a few plaintexts satisfy the algorithm of the one-way function of the MAP from (1) the fact that the MAP algorithm has the one to one correspondence ${ }^{[1]}$ and (2) the result of the cycling closure test of FEAL ${ }^{[12]}$, that has a very similar algorithm to this one-way function of the MAP.

\section{Concluding Remarks}

We studied into the method which improves FEAL algorithm against cryptanal$y$ sis based on the careful analysis of data randomizer. Since the round function $f$ of FEAL has an inverse function $\mathrm{f}^{-1}$, we studied an attack using the inverse function $\mathrm{f}^{-1}$ and key-independent intermediate messages. In this paper, we present an example of the application of our method to the study of ciphertext only attack for a one-way function that employs the mapping function called MAP. The algorithm of this MAP function is very similar to that of FEAL data randomizer from the view point of round function $f$.

Simulation results of the proposed attack have shown that the the computational complexity to obtain the plaintext of the one-way function of the MAP ${ }^{[1]}$ is decreased to $O\left(2^{32}\right)$ from $O\left(2^{64}\right)$. Then, the inverse function of this one-way function can be practically breakable by the proposed attack. 
15.7 hours are required to compute our example, with $\mathrm{C}$ program on a workstation(SUN-SPARCstation 2). There are three computed plaintext in this example. The obtained plaintext is not unique. But we can restrict the plaintext within narrow limits.

\section{$5 \quad$ References}

[1] Miyaguchi, S. : "CRITERIA FOR THE STRENGTH OF ENCIPHERMENT AND STANDARDIZATION FOR CRYPTOGRAPHIC TECH-

NIQUES", Proc. of the 1986 Symposium on Cryptography and Information Security, (1986).

[2] Shimizu, A., Miyaguchi, S.:"Fast Data Encipherment Algorithm FEAL", Advances in Cryptology - EUROCRYPT'87, p267-278 (1987).

[3] E. Biham, A. Shamir:" Differential Cryptanalysis of Feal and N-Hash ", Advances in Cryptology - EUROCRYPT'91, p1-16 (1991).

[4] H. Gilbert, G. Chasse :" A STATISTICAL ATTACK OF THE FEAL-8 CRYPTOSYSTEM", Advances in Cryptology - CRYPTO'90, p22-33 (1990). [5] A. TARDY-CORFDIR, H. GILBERT:" A KNOWN PLAINTEXT ATTACK OF FEAL-4 AND FEAL-6", Advances in Cryptology - CRYPTO'91, p172-182 (1991).

[6] Kaneko, T. :" A known plaintext cryptanalytic attack on FEAL-4" ,IEICE Tech. Report, ISEC91-25 (1991) (in Japanese).

[7] Matsui, M., Yamagishi, A.:"A Study on Known Text Attack of InvolutionType Cryptosystems",IEICE Tech. Report, ISEC91-26 (1991) (in Japanese).

[8] Tsujii, S., Kasahara, M. :" Cryptography and Information Security" ShohKohdo (1990) (in Japanese).

[9] Matsui, K. :" Introduction of Cryptoanalysis" Morikita Publishing Co. (1990) (in Japanese).

[10] Tsunoo, Y., Okamoto, E., Doi, H. :"Analytical Known Plain-text Attack for FEAL-4 and Its Improvement" , Proc. of the 1993 Symposium on Cryptography and Information Security, SCIS93-3A (1993) (in Japanese).

[11] Okamoto, E.:" An Introduction to Theory of Cryptology " Kyoritsu Publishing Co. (1993) (in Japanese).

[12] Kazuo, N.:"Cycling closure test of FEAL" IEICE Tech. Report, ISEC9050 (1990) (in Japanese). 